\title{
Toward more precise photometric redshift estimation
}

\author{
O. Vince ${ }^{1}$ and I. Csabai ${ }^{2}$ \\ ${ }^{1,2}$ ELTE University of Budapest, Pazmany P. Setany 1/A Budapest, Hungary \\ ${ }^{1}$ email: vince@complex.elte.hu \\ ${ }^{2}$ email: csabai@complex.elte.hu
}

\begin{abstract}
We investigate how morphological information of galaxies help us to improve photometric redshift estimation. Using a catalog of morphologically classified bright galaxies derived from the Third Data Release of the Sloan Digital Sky Survey, the statistical properties of different photometrical parameters as a function of morphology are examined. Parameters that best correlates with morphology are used as an additional information to better estimate the redhift. Improvements of several percent are obtained.
\end{abstract}

Keywords. morphology, redshift, SDSS

\section{Introduction}

To study galaxy evolution we need to acquire a large number of redshift sample that cover a substantial volume of the Universe. SDSS is one of the largest sky survey today that provide us with several millions of photometrically and about half million of spectroscopically observed objects. Although more precise, spectroscopic redshift sample cover extensively smaller volume in the space. Thus, different techniques are used to determine the galaxy redshift from photometrical observations. Basically, these techniques can be divided in two classes - SED-fitting method and Empirical method.

In this paper we give a short recall of each of these methods together with their shortcomings and virtue and investigate how morphological information help us to improve the accuracy of the photometric redshift estimation.

\section{Different techniques for photometrical redshift estimation}

SED-fitting method starts with a library of template spectra (empirical or modelled). Template spectra are corrected for redshift and Galactic extinction first and template colors are calculated thereafter by convolving template spectra with filter functions. Template colors are compared to the observed one minimizing the merit function $\chi^{2}(z, T)$.

On the other hand, Empirical method starts with a galaxy sample with known spectroscopical redshift $z_{s p}$, and magnitude m. Relationship $z_{s p}=z_{s p}(m)$ is determined and used as a calibration curve to get photometric redshift $z_{p h}$.

Details for both methods, advantages/disadvantages and about limitations can be found in Csabai et al. (2003) whereas more information about sources of uncertainties in Benitez (2000).

\section{Method}

In this paper, we try to improve photometric redshift estimation by reducing the redshift-morphology degeneracy (higher redshift early-type galaxies may have the same 
Table 1. Dissimilarity between spectroscopically and photometrically determined redhsift (see text for details)

\begin{tabular}{lcc}
\hline param & $\sigma_{r m s}$ & $\sigma_{r e l}$ \\
\hline $\mathrm{z}$ & 0.0298 & 0.0257 \\
$\mathrm{CIu}$ & $0.0295(1)$ & $0.0254(1)$ \\
$\mathrm{CIg}$ & $0.0289(3)$ & $0.0249(3)$ \\
$\mathrm{CIr}$ & $0.0291(2)$ & $0.0251(2)$ \\
$\mathrm{CIi}$ & $0.0292(2)$ & $0.0252(2)$ \\
$\mathrm{CIz}$ & $0.0293(2)$ & $0.0252(2)$ \\
fracDevu & $0.0296(1)$ & $0.0255(1)$ \\
fracDevg & $0.0295(1)$ & $0.0255(1)$ \\
fracDevr & $0.0295(1)$ & $0.0255(1)$ \\
fracDevi & $0.0295(1)$ & $0.0255(1)$ \\
fracDevz & $0.0295(1)$ & $0.0254(1)$ \\
\hline
\end{tabular}

color as less distant late-type). We have correlated all photometrical parameters from the SDSS catalog with visually determined morphological parameter, T, obtained by Nakamura et al. (2003). We found that concentration index (CI) and weight of deVaucouleurs component in best composite model (fracDev) best correlates with T. These two parameters are used further as a morphological information to break the degeneracy in the Empirical method for photometric redshift estimation.

\section{Result and Conclusion}

Result of this exercise is presented in Table 1. In the first column, names of the photometric parameters are given for all five filters used in the SDSS catalog $(\mathrm{u}, \mathrm{g}$, $\mathrm{r}$, i and z) designated with the last letter. Second and third columns are the uncertainties of the photometric redshift estimation given by $\sigma_{r m s}^{2}=<z_{s p}-z_{p h}>$ and $\sigma_{r e l}^{2}=<\left(z_{s p}-z_{p h}\right) /\left(1+z_{s p}\right)>$ respectively. In the parentheses, improvements of the photometric redshift estimation are given in percentage when the morphological information is used in the Empirical method. Exception is the first row where no morphological information is used in the calculation. As it can be seen, improvement of only several percent are obtained.

We also tried to improve photometric redshift estimation by using directly the morphological parameter $\mathrm{T}$ and about two percent of improvement is obtained. Somewhat higher improvement (4 percent) we got when the number of morphological parameters in the calculation is increased by one. Leaving out photometrical parameters observed in the $\mathrm{u}$ and $\mathrm{z}$ band where measurement errors are larger just slightly improves the photometric redshift (2 percent). Thus, we may conclude that the usage of morphological information in the Empirical method negligible improves photometric redshift estimation.

\section{References}

Benitez 2003, ApJ, 536, 571

Csabai, I., Budavari, T., Connoly, A., Szalay, A., Gyory, Zs., Benitez, N., Annis, J., Brinkmann, J., Eisentein, D., Fukugita, M., Gunn, J., Knet, S., Lupton, R., Nichol, R. \& Stoughton, C. $2003, A J 125,580$

Nakamura, O., Fukugita, M., Yasuda, N., Loveday, J., Brinkmann, J., Schneider, P.D., Shimasaku, K. \& SubbaRao, M. 2002, AJ 125, 1682 